\title{
Une nouvelle série d'analyse des cheveux de Napoléon confirme une exposition chronique à l'arsenic
}

\section{A new serie of hair analysis from Napoleon confirms chronic exposure to arsenic}

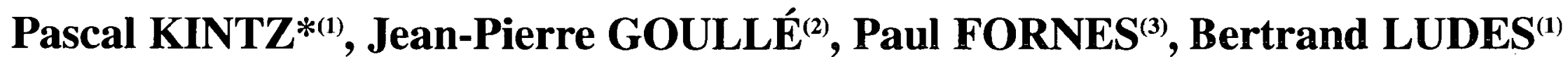

(1) Institut de Médecine Légale, 11, rue Humann - 67000 STRASBOURG (2) Laboratoire de Pharmacologie et de Toxicologie, CHG - LE HAVRE

(3) Médecine Légale, Faculté de Médecine Cochin-Port Royal - PARIS

* Auteur à qui adresser la correspondance : Docteur Pascal KINTZ, Institut de Médecine Légale, 11, rue Humann 67000 STRASBOURG - Tél : +33 (0)390243349 - Fax : +33 (0)390243362

E-mail : pascal.kintz@wanadoo.fr

(Reçu le 24 octobre 2001 ; accepté le 28 novembre 2001)

\section{RÉSUMÉ}

Cancer de l'estomac ou empoisonnement par l'arsenic? Décédé le 5 mai 1821 sur l'île de Sainte Hélène, Napoléon Bonaparte est l'objet de nombreuses spéculations quant à la cause exacte de sa mort.

Dès 1960, le laboratoire de recherche de la centrale nucléaire de Harwell, avec l'aide de l'Université de Glasgow mettait en évidence de l'arsenic dans les cheveux de Napoléon, à une concentration supérieure à la normale. Ces résultats étaient confirmés en 1995 par le FBI américain.

En avril 2000, lors d'une réunion au Sénat, ces résultats ont été contestés par des toxicologues français.

Afin de compléter les analyses précédentes, le $\mathrm{Dr} B e n$ Weider, Président de la Société napoléonienne internationale a remis à l'Institut de Médecine Légale de Strasbourg 5

\section{SUMMARY}

Since 1964, and the presentation of the results of the hair analysis of Napoleon by the Harwell Nuclear Research laboratory of the University of Glasgow demonstrating high arsenic content, there has been a great controversy about the final conclusions, involving a criminal poisoning. More recently, the US Federal Bureau of Investigation concluded that the amount of arsenic present in the submitted hairs is consistent with arsenic poisoning. On the opposite, several authors demonstrated no evidence of chronic arsenic poisoning or, if Napoleon was poisoned by arsenic, it may have come not from deliberate administration but from his wallpaper. Our laboratory received 5 different specimens strands of hair attributed to Napoleon that were tested by 
échantillons de cheveux de l'Empereur, authentifiés par ses soins. Il s'agit d'un échantillon prélevé par le comte Las Cases le 16 mars 1816, de 2 échantillons rasés le 6 mai 1821 et donnés à Lady Holland et à l'abbé Vignali et enfin de 2 autres échantillons rasés le 6 mai 1821 et donnés à Abram Noverraz et à Louis Marchand.

Après décontamination par de l'acétone et de l'eau chaude, les cheveux pesant entre 0,5 et 2,2 mg, ont été hydrolysés par de la soude 1,00 N, puis l'homogénat a été neutralisé par de l'acide nitrique 1,03 N. L'arsenic a été dosé par spectrophotométrie d'absorption atomique, en mode pyrolytique, par la méthode des ajouts dosés.

Selon les échantillons analysés, les concentrations d'arsenic ont varié de 6,99 $\mathrm{ng} / \mathrm{mg}$ à $38,53 \mathrm{ng} / \mathrm{mg}$. La physiologie humaine accepte des concentrations capillaires en arsenic, variant de 0,1 à $1,0 \mathrm{ng} / \mathrm{mg}$. L'analyse des cheveux de Napoléon montre donc sans aucune ambiguïté une exposition répétée à l'arsenic.

\section{MOTS-CLÉS}

Napoléon, cheveux, arsenic.

\section{Introduction}

Napoléon Bonaparte est mort sur l'île de Sainte Hélène le 5 mai 1821. Depuis la fin du $20^{\text {ème }}$ siècle, un débat international a pris place sur les causes exactes de son décès, comme en attestent les nombreuses publications, sous forme d'articles ou de livres, sur le sujet. La thèse officielle retient le cancer de l'estomac, compliqué par une hémorragie gastrique, favorisée par des grandes quantités de calomel administré le jour précédant le décès.

Or, dès 1964, le laboratoire de recherche nucléaire Harwell (Université de Glasgow), dans la banlieue de Londres, mettait en évidence par activation neutronique dans les cheveux de l'Empereur des concentrations importantes d'arsenic, très évocatrices d'une administration prolongée. Dans leur conclusion, les auteurs parlaient alors d'empoisonnement chronique (1). Ces résultats étaient repris récemment par Weider et Fournier (2) pour confirmer la thèse de l'empoisonnement.

Plusieurs cheveux attribués à l'Empereur ont été analysés dans divers laboratoires internationaux, dont la plupart mettaient en évidence des concentrations en arsenic très supérieures aux concentrations physiologiques. En 1998, Hindmarsh et Corso (3) publient un compendium des résultats (environ une vingtaine) des analyses de cheveux réalisées jusqu'à ce jour.

Dans le même temps, la thèse de l'empoisonnement était combattue par les tenants de la version officielle, avançant les arguments suivants : les cheveux n'appartiennent pas à Napoléon, il y a des contaminations externes, l'arsenic provient du papier peint de la graphite furnace atomic absorption spectophotometry. Arsenic was in the range 6.99 to $38.53 \mathrm{ng} / \mathrm{mg}$, clearly demonstrating chronic and long-term exposure. An extensive decontamination procedure permitted us to exclude external contamination.

\section{KEY-WORDS}

Napoleon, hair, arsenic.

chambre de l'Empereur ou d'un dégagement par le charbon du chauffage, l'eau de boisson était contaminée ou encore l'arsenic est le résultat de soins de conservation (4-6).

C'est dans ce climat particulier où se disputaient l'histoire et la toxicologie que notre laboratoire a reçu en septembre 2000, des mains du Docteur Ben Weider, Président de la Société Napoléonienne Internationale, 5 mèches de cheveux attribuées à l'Empereur aux fins d'analyses toxicologiques, en particulier pour mesurer la concentration en arsenic.

\section{Matériel et méthode Prélèvements de cheveux}

Les échantillons suivants ont été soumis au laboratoire de Toxicologie de l'institut de Médecine Légale de Strasbourg :

- Cheveux identifiés «Las Cases», coupés par Santini le 16 octobre 1816 et conservés par Las Cases. Certains de ces cheveux ont été donnés à Monsieur William Fraser (Dehli, Inde) dans une enveloppe. Cette enveloppe était la propriété de Monsieur Troubetskoy (USA) qui l'a remise au Docteur Hamilton Smith. Nous avons analysé 2 cheveux.

- Cheveux identifiés «abbé Vignali», coupés le 6 mai 1821 et conservés par l'abbé (Figure 1).

- Cheveux identifiés «lady Holland», coupés le 6 mai 1821 et conservés dans une boîte en or, donnée à lady Holland. La mèche était attachée au centre d'un médaillon (Figure 2). 


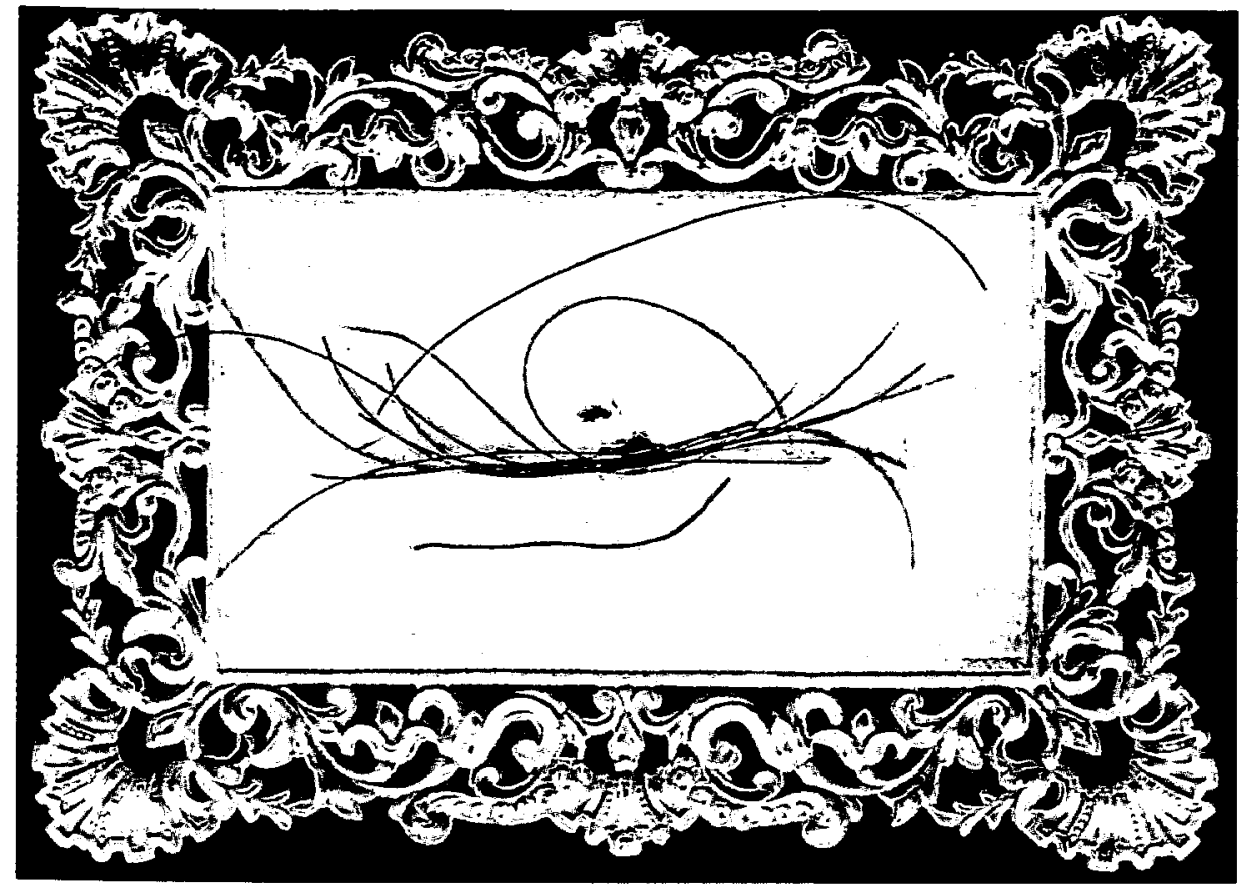

Figure 1 : Photo représentant la mèche de cheveux de Napoléon dite "Abbé Vignali".

- Cheveux identifiés «Abram Noverraz», coupés le 6 mai 1821 et appartenant au musée d'Arenenberg (Suisse) qui nous a donné 9 cheveux. Ces cheveux sont les plus longs jamais analysés (certains mesuraient $9 \mathrm{~cm}$ ).

- Cheveux identifiés «Louis Marchand», coupés le 6 mai 1821 et appartenant à Monsieur Jean Tranié qui nous a donné 3 cheveux.

\section{Analyse toxicologique}

Les cheveux ont été au préalable décontaminés par un passage successif dans les bains suivants : acétone ( $5 \mathrm{ml}, 2 \mathrm{~min}$ ), eau chaude ( $5 \mathrm{ml}, 2 \mathrm{~min}$ ) et enfin acétone $(5 \mathrm{ml}, 2 \mathrm{~min})$, avec à chaque fois agitation vigoureuse.

Après pesée, les cheveux $(0,5$ à $2,2 \mathrm{mg})$ ont été hydrolysés dans $60 \mu \mathrm{l}$ de $1 \mathrm{M} \mathrm{NaOH}$ au bloc chauffant $\left(90^{\circ} \mathrm{C}\right)$ pendant $30 \mathrm{~min}$. La neutralisation, avec un léger excès acide, est effectuée par $60 \mu \mathrm{l}$ de $1,03 \mathrm{M}$ d'acide nitrique. Avant la mesure spectrophotométrique, $400 \mu \mathrm{l}$ de modificateur de matrice (nitrate de nickel à $6 \mathrm{~g} / \mathrm{l}$ de nickel) ont été ajoutés.

Le dosage de l'arsenic total a été réalisé en mode ajouts dosés, par four pyrolytique, avec lecture à 193,7 nm (fente à $0,5 \mathrm{~nm}$ ) sur un système d'absorption atomique Varian SpectrAA-800 avec correction Zeeman. Ces mesures ont été faites au Centre Hospitalier du Havre, chez le Dr Jean-Pierre Goullé.

\section{Résultats et discussion}

La correction Zeeman a permis d'éliminer les absorptions non-spécifiques.

A partir d'une prise d'essai de $2 \mathrm{mg}$ de cheveux, la

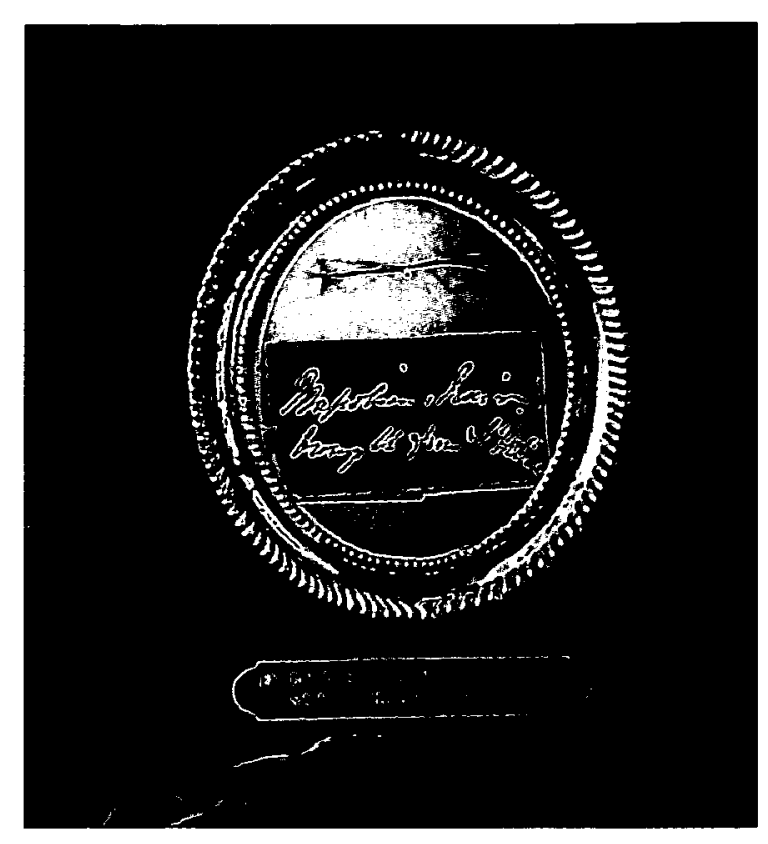

Figure 2: : Photo représentant le médaillon contenant la mèche de cheveux de Napoléon dite "lady Holland".

linéarité a été vérifiée de 0,5 à $120 \mathrm{ng}$ d'arsenic par mg de cheveux. Dans ces conditions, la limite de détection de la machine a été de $0,11 \mathrm{ng} / \mathrm{mg}$. La répétabilité, évaluée sur 20 mesures consécutives, était de 9,7\%.

Les résultats des investigations toxicologiques sur les 5 échantillons de cheveux figurent dans le Tableau I. Compte tenu des faibles quantités de cheveux remis aux toxicologues, nous avons choisi de ne pas faire d'analyse segmentaire, qui aurait eu comme risque de nous faire travailler dans le bruit de fond de l'appareil. Il nous a paru délicat d'utiliser une prise d'essai de quelques $\mu \mathrm{g}$ pour établir une chronologie temporelle de l'intoxication, d'autant qu'il est toujours difficile d'interpréter les concentrations en fonction de la vitesse de pousse, qui est ici, par définition, non mesurable. De même, il ne nous a pas été possible d'utiliser l'ICP/MS, plus spécifique, cette technologie nécessitant de grande quantité de matériel biologique ( $>200 \mathrm{mg}$ ).

La littérature internationale la plus récente (7) accepte des concentrations physiologiques d'arsenic dans les cheveux entre 0,1 et $1,0 \mathrm{ng} / \mathrm{mg}$ et considère que des concentrations supérieures sont très en faveur d'une exposition répétée. Dans ces conditions, tous les échantillons de cheveux attribués à l'Empereur apparaissent comme positifs et confirment donc une intoxication à long terme par l'arsenic.

Il n'est pas du ressort des analystes de discuter l'éventuel caractère criminel de cette intoxication.

Par contre, il convient de confirmer l'efficacité de notre procédure de décontamination, afin d'éliminer toute déposition sur la tige pilaire d'arsenic exogène. Une contamination externe pourrait exister par l'utilisation de cosmétiques avant le décès. Selon toutes les informations fournies par les historiens, il ne semble pas que l'Empereur utilisait des soins capillaires à base d'arse- 
Tableau I : Concentrations en arsenic dans les cheveux de Napoléon Bonaparte.

\begin{tabular}{|c|c|c|}
\hline Cheveux & $\begin{array}{c}\text { Masse analysée } \\
\text { (mg) }\end{array}$ & $\begin{array}{c}\text { Concentration en arsenic } \\
\text { (ng/mg) }\end{array}$ \\
\hline Las Cases & 0,5 & 7,43 \\
\hline Abbé Vignali & 2,0 & 15,50 \\
\hline Lady Holland & 1,2 & 38,53 \\
\hline Abram Noverraz & 2,2 & 6,99 \\
\hline Louis Marchänd & $\vdots 0,5$ & 15,20 \\
\hline
\end{tabular}

nic. De même, il est possible d'exclure une positivité due aux soins de conservation ou une contamination par la terre pendant les 19 premières années d'inhumation (les toxicologues se souviendront ici des polémiques de l'affaire Marie Besnard), puisque les cheveux ont été prélevés au moment du décès.

L'efficacité de notre procédure de décontamination a été prouvée par une simple expérience de contamination sévère. Une mèche de cheveux (témoin négatif) a été incubée pendant 4 heures dans une solution aqueuse d' $\mathrm{As}_{2} \mathrm{O}_{3}$ à $10 \mathrm{mg} / \mathrm{l}$. Avant contamination, la concentration en arsenic était à $0,31 \mathrm{ng} / \mathrm{mg}$. L'analyse après contamination mais sans décontamination met en évidence de l'arsenic à $9,86 \mathrm{ng} / \mathrm{mg}$, qui est éliminé lorsque l'on effectue une phase triple de décontamination (séquence acétone/eau chaude/acétone), puisque la concentration revient alors à $0,34 \mathrm{ng} / \mathrm{mg}$.

Une réponse positive en arsenic pourrait également avoir pour origine : l'utilisation de préparations pharmaceutiques contenant de l'arsenic (aucun historien $\mathrm{n}$ 'a $\mathrm{pu}$ fournir une quelconque information sur ce sujet), une contamination de l'eau de boisson de
Longwood (cette eau a été analysée par Hindmarsh et Corso de nos jours - la concentration en arsenic était inférieure à $0,002 \mathrm{ppm}$ ), une contamination par le papier peint (or celui-ci a été changé en 1819, ce qui ne permet donc pas d'expliquer les concentrations impor-' tantes dans la mèche Las Cases, datant de 1816) et enfin l'inhalation de résidus d'arsenic provenant de la combustion de charbon (seul l'Empereur présentait des signes d'intoxication et pas les autres habitants de l'île). Dans ces conditions, il apparait comme raisonnable sur le plan scientifique de ne retenir que l'intoxication par voie orale.

Enfin, les différences de concentrations entre les divers échantillons, pourtant recueillis à la même date, pourraient s'expliquer par des longueurs différentes des cheveux, des phases de croissance différentes (seuls les cheveux en phase anagène incorporent les xénobiotiques, par comparaison avec les phases catagène et télogène) et enfin une localisation anatomique variable du site de prélèvement.

\section{Conclusion}

L'analyse par spectrophotométrie d'absorption atomique électrothermique de 5 prélèvements de cheveux attribués à Napoléon Bonaparte a permis de mettre en évidence une exposition majeure à l'arsenic. Les concentrations mesurées sont compatibles avec une intoxication chronique. Notre procédure efficace de décontamination a permis de minimiser le problème de la contamination externe. Idéalement, afin de compléter définitivement la validation de cette expertise, il conviendrait d'authentifier les cheveux de l'Empereur et de mesurer la concentration en arsenic de quelques échantillons de cheveux prélevés à cette époque.

\section{Références}

1. Forshufvud S., Smith H., Wassen A. Napoleon's illness 1816-1821 in the light of activation analyses in hairs from various dates. Arch. Toxicol. $1964 ; 20: 210-9$.

2. Weider B., Fournier J.H. Activation analyses of authenticated hairs of Napoleon Bonaparte confirm arsenic poisoning. Am. J. Forensic Med. Pathol. 1999 ; 20 : 37882.

3. Hindmarsh J.T., Corso P.F. The death of Napoleon Bonaparte : a critical review of the cause. J. History Med. 1998 ; 53 : 201-18.

4. Lewin P.K., Hancock G.V., Voynovich P. Napoleon Bonaparte - no evidence of chronic arsenic poisoning. Nature $1982 ; 299: 627-8$.
5. Jones D.E.H., Ledingham K.W.D. Arsenic in Napoleon's wall paper. Nature $1982 ; 299: 266-7$.

6. Corso P.F., Hindmarch J.T., Dello Stritto F. The death of Napoleon. Am. J. Forensic Med. Pathol. 2000 ; 21 : 300-5.

7. Baselt R.C. Arsenic. In : Baselt R.C. ed. Disposition of toxic drugs and chemicals in man. Fifth edition. Foster City : Chemical Toxicology Institute, 2000 ; 61-4. 